\title{
AN IDENTITY FOR COCYCLES ON COSET SPACES OF LOCALLY COMPACT GROUPS
}

\author{
H.KUMUDINI DHARMADASA AND WILLIAM MORAN
}

\begin{abstract}
We prove here an identity for cocycles associated with homogeneous spaces in the context of locally compact groups. Mackey introduced cocycles ( $\lambda$-functions) in his work on representation theory of such groups. For a given locally compact group $G$ and a closed subgroup $H$ of $G$, with right coset space $G / H$, a cocycle $\lambda$ is a real-valued Borel function on $G / H \times G$ satisfying the cocycle identity

$$
\lambda(x, s t)=\lambda(x . s, t) \lambda(x, s), \quad \text { a.e. } x \in G / H, s, t \in G,
$$

where the "almost everywhere" is with respect to a measure whose null sets pull back to Haar measure null sets on $G$. Let $H$ and $K$ be regularly related closed subgroups of $G$. Our identity describes a relationship between cocycles for $G / H^{x}, G / K^{y}$ and $G /\left(H^{x} \cap K^{y}\right)$ for almost all $x, y \in G$. This also leads to an identity for modular functions of $G$ and the corresponding subgroups.
\end{abstract}

\section{Introduction and Statement of Results}

The aim of this paper is to prove an identity for cocycles (Mackey's $\lambda$ functions). The need for this identity arose in connection with problems on induced representations to be discussed in a later publication. Let $G$ be a separable locally compact group and $H$ a closed subgroup of $G$. In his treatment of Induced Representation on Locally Compact Groups ([4]), Mackey introduced the concept of a cocycle $\lambda$ as a real valued positive function on $(G / H) \times G$ satisfying certain identities (see sec.2.1). Most importantly, such cocycles are associated with quasi-invariant measures; to each such cocycle there is a quasi-invariant measure $\mu$ on $G / H$, so that the Radon-Nikodym derivative of the translated measure $\mu . s$ with respect to $\mu$ is $\lambda(\cdot, s)$ for all $s \in G$. Once $\lambda$ is specified, this measure is unique up to a positive scalar multiple. The basic properties of these functions are well-established in the literature (cf. $[1,4,5]$ ).

To state our results, we will need some concepts and notations. Let $\lambda_{H}$ denote a cocycle corresponding to the homogeneous space $G / H$ and let $H^{x}=x^{-1} H x$, for $x \in G$. Let $\Delta_{H}$ be the modular function of the group $H$. Closed subgroups $H$ and $K$ of $G$ are said to be regularly related if the double coset space $H \backslash G / K$ is a standard Borel space (cf.[4]). We note that the double coset space formed by the right action of the diagonal subgroup $\Lambda=\{(x, x): x \in G\}$ of $G \times G$ on the coset space $(H \times K) \backslash(G \times G)$ (that is, $H \times K \backslash G \times G / \Lambda)$ is identified with $H \backslash G / K$ by the map $(x, y) \mapsto x y^{-1}$. The regularly related property for $H$ and $K$ is equivalent to this action being smooth $([4])$.

Our main result provides a link between cocycles for conjugates of regularly related subgroups. We will abuse notation and assume that the cocycle $\lambda_{H}(s, t)$ is actually defined on $G \times G$ and constant on the right cosets of $H$, rather than on $(G / H) \times G$.

Theorem 1. Let $G$ be a separable locally compact group and $H, K$ closed subgroups of $G$. If $H$ and $K$ are regularly related, then for each double coset $D(x, y)=(H \times K)(x, y) \Lambda$, there is a quasi-invariant measure

Date: April, 24, 2016.

2010 Mathematics Subject Classification. 43A65, 22D30, 43A15.

Key words and phrases. Separable locally compact group, quasi-invariant measure, modular function, $\lambda-$ function. 
$\mu_{x, y}$ on $G /\left(H^{x} \cap K^{y}\right), x, y \in G$, and a corresponding cocycle $\lambda_{H^{x} \cap K^{y}}$ such that

$$
\lambda_{H^{x}}\left(t s^{-1}, s\right) \lambda_{K^{y}}\left(t s^{-1}, s\right) \lambda_{H^{x} \cap K^{y}}\left(t, s^{-1}\right)=1, \quad(s, t \in G, \text { a.e. }(x, y) \in(G \times G) /(H \times K)) .
$$

Moreover, $\lambda_{H^{x} \cap K^{y}}(t, s)$ is defined everywhere and continuous on $\left(G /\left(H^{x} \cap K^{y}\right)\right) \times G$.

Theorem 1 leads to an identity relating the modular functions corresponding to the subgroups involved:

Corollary 1. For $(x, y) \in G \times G$ such that (1) holds, and for $s \in H^{x} \cap K^{y}$,

$$
\frac{\Delta_{H^{x}}(s) \Delta_{K^{y}}(s)}{\Delta_{G}(s) \Delta_{H^{x} \cap K^{y}}(s)}=1
$$

A necessary and sufficient condition for the existence of an invariant measure on a quotient group $G / H$ of $G$ is that $\Delta_{H}(x)=\Delta_{G}(x)$ for all $x \in H$. We say that $G \supset H$ is comodular if this happens. Now we have the following straightforward consequence of Corollary 1.

Corollary 2. Let $H$ and $K$ be regularly related closed subgroups of a separable locally compact group $G$ and $(x, y) \in G \times G$ such that the identity (1) holds. If $G \supset H$ is comodular, then $K \supset\left(H^{z} \cap K\right)$ is comodular for almost all $z \in G$.

\section{Preliminaries on COCYCles}

To avoid measure theoretic complications, we assume throughout that $G$ is a locally compact separable group and $H$ a closed subgroup. We denote the right-invariant Haar measure on $G$ by $\nu_{G}$, with $e$ denoting the identity of the group. The canonical mapping from $G$ to the set of right-cosets $G / H$ is denoted by $p_{H}$. Throughout this section, $X$ denotes the set of right cosets $G / H$ of $H$ with the standard right action of $G$. A left action by any other (closed) subgroup $K$ of $G$ gives rise to orbits in one-to-one correspondence with the double cosets $H \backslash G / K$, and the stabilizer of $H x \in X$ under the action of $K$ is $H^{x} \cap K$.

We briefly list the key results on cocycles, quasi-invariant measures and related concepts from our perspective. The reader is referred to $[1,4,5]$.

- There is a regular Borel section $B \subset G$; that is, a Borel set $B$ that intersects each right $G$ coset in exactly one point such that $\left(p_{H}^{-1}\left(p_{H}(K)\right)\right) \cap B$ has a compact closure for each compact subset $K$ of $G$.

- A strictly positive, real-valued continuous function $\rho_{H}$ exists on $G$ satisfying

$$
\rho_{H}(h x)=\left(\Delta_{H}(h) / \Delta_{G}(h)\right) \rho_{H}(x), \quad(x \in G, h \in H) .
$$

- Such a $\rho$-function gives rise to a unique Borel cocycle $\lambda_{\rho}$ on $X \times G$ such that

$$
\lambda_{\rho}\left(p_{H}(s), y\right)=\frac{\rho(s y)}{\rho(s)}, \quad(s, y \in G)
$$

with the properties:

(a) for all $x \in X$ and $s, t \in G, \quad \lambda_{\rho}(x, s t)=\lambda_{\rho}(x . s, t) \lambda_{\rho}(x, s)$;

(b) for all $h \in H, \lambda_{\rho}\left(p_{H}(e), h\right)=\Delta_{H}(h) / \Delta_{G}(h)$;

(c) for $t \in G, \lambda_{\rho}\left(p_{H}(e), t\right)$ is bounded on compact sets as a function of $t$; For $x, t \in G$ and for almost all $v \in G / H, \lambda_{H^{x}}\left(x^{-1} v, t\right)=\lambda_{H}(v, t)$.

- For each $\rho$-function on $G$ there is a quasi-invariant measure $\mu$ on $X$ such that, for all $y \in G$, the corresponding cocycle $\lambda_{\rho}$ has the property that $\lambda_{\rho}(\cdot, y)$ is a Radon-Nikodym derivative of the translation measure $\mu . y$ with respect to the measure $\mu$. 
- For $x \in G$ let $\dot{x}=p_{H}(x)$. If $\mu$ denotes the quasi-invariant measure corresponding to the function $\rho$ then

$$
\int_{G} f(x) \rho(x) d \nu_{G}(x)=\int_{X} \int_{H} f(h x) d \nu_{H}(h) d \mu(\dot{x}), \quad\left(f \in C_{00}(G)\right) .
$$

where $C_{00}(G)$ denotes the continuous functions on $G$ with compact support. We write $\mu \succ \lambda$ to mean that for all $y \in G, \lambda(\cdot, y)$ is the cocycle that is the Radon-Nikodym derivative of the translate $E \mapsto \mu([E] y)$ with respect to $\mu$. The following facts on $\lambda$ and corresponding $\rho$-functions can be found in many places in the literature (see, for example, $[4,1]$ ).

There are quasi-invariant measures on $X$, any two of which are absolutely continuous. Null sets for such measures are exactly those sets $E$ for which $p_{H}^{-1}(E)$ has Haar measure zero. The relations $\mu \succ \lambda$ and $\lambda=\lambda_{\rho}$ between quasi-invariant measures, cocycles, and $\rho$-functions have the following properties:

(i) Every cocycle is of the form $\lambda_{\rho} ; \lambda_{\rho_{1}}=\lambda_{\rho_{2}}$ if and only if $\rho_{1} / \rho_{2}$ is a constant.

(ii) For every cocycle there is a quasi-invariant measure $\mu$ such that $\mu \succ \lambda$; if $\mu_{1} \succ \lambda$ and $\mu_{2} \succ \lambda$ then $\mu_{1}$ is a constant multiple of $\mu_{2}$.

(iii) For every quasi-invariant measure $\mu$ there is a cocycle $\lambda$ such that $\mu \succ \lambda$. If $\mu \succ \lambda_{1}$ and $\mu \succ \lambda_{2}$ then, for all $t, \lambda_{1}(\cdot, t)=\lambda_{2}(\cdot, t)$ a.e.

(iv) If $\mu \succ \lambda_{\rho_{1}}$ and $\mu \succ \lambda_{\rho_{2}}$ then $\rho_{1} / \rho_{2}$ is constant almost everywhere.

\section{Proofs of the Results}

First we recall some standard results on disintegration of measures. Let $X$ be a separable locally compact space supporting a finite measure $\mu$ and let $R$ be an equivalence relation on $X$ where $r(x)$ is the equivalence class containing $x$. The relation $R$ is measurable if there exists a countable family $E_{1}, E_{2}, \ldots$ of subsets of $X / R$ such that $r^{-1}\left(E_{i}\right)$ is measurable for each $i$ and such that each point in $X / R$ is the intersection of the $E_{i}$ containing it (cf. $\left.[4,2]\right)$.

It is well known (see, for example, [4], p.124, Lemma 11.1) that the measure $\mu$ is decomposable as an integral over $X / R$ of measures $\mu_{y}$ concentrated on the equivalence classes.

If $\tilde{\mu}$ is the "push-forward" measure on $X / R$ from the measure $\mu$ on $X$, i.e. $\tilde{\mu}(E)=\mu\left(r^{-1}(E)\right)$, then for each $y$ in $X / R$ there exists a finite Borel measure $\mu_{y}$ on $X$ such that $\mu_{y}\left(X-r^{-1}(\{y\})\right)=0$ and

$$
\int f(y) \int g(x) d \mu_{y}(x) d \tilde{\mu}(y)=\int f(r(x)) g(x) d \mu(x),
$$

whenever $f \in L_{1}(X / R, \tilde{\mu})$ and $g$ is bounded and measurable on $X$. If $\mu$ is quasi-invariant then in the disintegration of $\mu$ in (2) above, $\mu_{y}$ is also quasi-invariant under the action of $G$ a.e. $y([4])$.

\section{Proof of Theorem 1.}

It is clear that if $H$ and $K$ are regularly related then the orbits of $G / H$ under the action of $K$ outside a set of measure zero form the equivalence classes of a measurable equivalence relation. The right action of the diagonal subgroup $\Lambda=\{(x, x): x \in G\}$ of $G \times G$ on the coset space $(G \times G) /(H \times K)$ has stabilizer $H^{x} \times K^{y} \cap \Lambda=(H \times K)^{(x, y)} \cap \Lambda$ at $(H x, K y)$, and the orbit of this point is the double coset $(H \times K)(x, y) \Lambda$. We write $\Upsilon$ for the set of all double cosets $(H \times K) \backslash G \times G / \Lambda \simeq H \backslash G / K$. As noted earlier, the regularly related property for $H$ and $K$ is equivalent to this orbit space being smooth ([4]). Writing $D(x, y)$ for the double coset to which $(x, y)$ belongs, for a fixed finite measure $\nu_{0}$ on $G \times G$ equivalent to Haar measure, we define a measure $\mu_{(H, K)}$ on $\Upsilon$ by $\mu_{(H, K)}(F)=\nu_{0}\left(D^{-1}(F)\right)$. Such a measure is called an admissible measure by Mackey. 
Fix a finite product measure $\nu_{0}=\nu_{1} \times \nu_{2}$ on $(G \times G)$ equivalent to Haar measure. Let $\mu_{H \times K}$ be the image of $\nu_{0}$ under $p_{H \times K}$ and $\mu_{H}, \mu_{K}$ the images of $\nu_{1}, \nu_{2}$ under $p_{H}$ and $p_{K}$ respectively. Let $\mu_{H, K}$ be an admissible measure in $\Upsilon$ corresponding to $\nu_{0}$.

For a function $f$ on $(G / H) \times(G / K)$ for which $\int_{G / H} \int_{G / K} f(x, y) d \mu_{H}(x) d \mu_{K}(y)$ is integrable, using the change of variables $x \mapsto x s$ and $y \mapsto y s$, we obtain

$$
\begin{array}{r}
\int_{G / H} \int_{G / K} f(x, y) d \mu_{H}(x) d \mu_{K}(y)=\int_{G / H} \int_{G / K} \lambda_{H}(x, s) \lambda_{K}(y, s) f(x s, y s) d \mu_{H}(x) d \mu_{K}(y) \\
=\int_{(G \times G) /(H \times K)} \lambda_{H}(x, s) \lambda_{K}(y, s) f(x s, y s) d \mu_{H \times K}(x, y) .
\end{array}
$$

For $(x, y) \in(G \times G) /(H \times K)$, write $r(x, y)=D\left(p_{H \times K}^{-1}(x, y)\right)$; this defines a measurable equivalence relation since $H$ and $K$ are regularly related. The measure $\mu_{H \times K}$ is disintegrated into an integral of measures $\mu_{x, y}$, where $D(x, y) \in \Upsilon$, with respect to the measure $\mu_{H, K}$ on $\Upsilon$. Also, each $\mu_{x, y}$ is a quasi-invariant measure on the orbit $r^{-1}(D(x, y))$ (cf. (2)). Using this disintegration, we have

$$
\begin{aligned}
\int_{(G \times G) /(H \times K)} \lambda_{H}(x, s) & \lambda_{K}(y, s) f(x s, y s) d \mu_{H \times K}(x, y) \\
= & \int_{D \in \Upsilon} \int_{\underline{t} \in \Lambda /(H \times K)^{(x, y)} \cap \Lambda} \lambda_{H}(x t, s) \lambda_{K}(y t, s) f(x t s, y t s) d \mu_{x, y}(\underline{t}) d \mu_{H, K}(D),
\end{aligned}
$$

where $(x, y)$ is a coset representative of the coset $D(x, y)$. Identifying the space $\Lambda /\left((H \times K)^{(x, y)} \cap \Lambda\right)$ with $G /\left(H^{x} \cap K^{y}\right)$, we can regard $\mu_{x, y}$ as a measure on $G /\left(H^{x} \cap K^{y}\right)$. Then we have

$$
\begin{aligned}
\int_{(G \times G) /(H \times K)} \lambda_{H}(x, s) \lambda_{K}(y, s) f(x s, y s) d \mu_{H \times K}(x, y) \\
=\int_{D \in \Upsilon} \int_{t \in G /\left(H^{x} \cap K^{y}\right)} \lambda_{H}(x t, s) \lambda_{K}(y t, s) f(x t s, y t s) d \mu_{x, y}(t) d \mu_{H, K}(D) .
\end{aligned}
$$

Changing variables $t \mapsto t s^{-1}$ in the integral on the right-hand side, we find that

$$
\begin{aligned}
& \int_{(G \times G) /(H \times K)} \lambda_{H}(x, s) \lambda_{K}(y, s) f(x s, y s) d \mu_{H \times K}(x, y) \\
& \quad=\int_{D \in \Upsilon} \int_{t \in G /\left(H^{x} \cap K^{y}\right)} \lambda_{H}\left(x t s^{-1}, s\right) \lambda_{K}\left(y t s^{-1}, s\right) f(x t, y t) \lambda_{H^{x} \cap K^{y}}\left(t, s^{-1}\right) d \mu_{x, y}(t) d \mu_{H, K}(D) .
\end{aligned}
$$

On the other hand, if we start with $\int_{(G \times G) /(H \times K)} f(x, y) d \mu_{H \times K}(x, y)$ and use disintegration, we have

$$
\begin{aligned}
& \iint_{(G \times G) /(H \times K)} f(x, y) d \mu_{H \times K}(x, y) \\
& =\int_{D \in \Upsilon} \int_{\underline{\underline{t}} \in \Lambda /(H \times K)^{(x, y)} \cap \Lambda} f(x t, y t) d \mu_{x, y}(t, t) d \mu_{(H, K)}(D) \\
& \quad=\int_{D \in \Upsilon} \int_{t \in G /\left(H^{x} \cap K^{y}\right)} f(x t, y t) d \mu_{x, y}(t) d \mu_{(H, K)}(D) .
\end{aligned}
$$

Now (4) and (5) yield

$$
\lambda_{H}\left(x t s^{-1}, s\right) \lambda_{K}\left(y t s^{-1}, s\right) \lambda_{H^{x} \cap K^{y}}\left(t, s^{-1}\right)=1, \quad\left(s \in G \text {, a.e. } t \in G /\left(H^{x} \cap K^{y}\right)\right) ;
$$

or, using the cocycle property (c) in Sec.2, 
(1) $\quad \lambda_{H^{x}}\left(t s^{-1}, s\right) \lambda_{K^{y}}\left(t s^{-1}, s\right) \lambda_{H^{x} \cap K^{y}}\left(t, s^{-1}\right)=1, \quad\left(s \in G\right.$, a.e. $\left.t \in G /\left(H^{x} \cap K^{y}\right)\right)$

for almost all $(x, y) \in(G \times G) /(H \times K)$. Fixing such an $(x, y)$ in $(G \times G) /(H \times K)$, and invoking continuity of $\lambda_{H}$ and $\lambda_{K}$, we see that (1) is true for all $t \in G /\left(H^{x} \cap K^{y}\right)$. Furthermore, (1) implies that $\lambda_{H^{x} \cap K^{y}}(t, s)$ is defined everywhere and continuous on $\left(G /\left(H^{x} \cap K^{y}\right)\right) \times G$.

Proof of Corollary 1. Setting $t=s$ in (1) and using the property (a) of cocycles in Sec. 2.1, we obtain

$$
\lambda_{H^{x}}(e, s) \lambda_{K^{y}}(e, s)=\lambda_{H^{x} \cap K^{y}}(e, s) .
$$

Now we use the property (b) of cocycles in Sec 2.1 to obtain

$$
\frac{\Delta_{H^{x}}(s)}{\Delta_{G}(s)} \frac{\Delta_{K^{y}}(s)}{\Delta_{G}(s)}=\frac{\Delta_{H^{x} \cap K^{y}}(s)}{\Delta_{G}(s)}
$$

This leads to the required equality

$$
\frac{\Delta_{H^{x}}(s)}{\Delta_{G}(s)} \frac{\Delta_{K^{y}}(s)}{\Delta_{H^{x} \cap K^{y}}(s)}=1
$$

\section{Remarks:}

- We emphasise that the result is an almost everywhere statement on the product space $G / H \times G / K$. If $H=K$, the diagonal $\{(x, x): x \in G / H\}$ will normally have zero measure. Indeed, if it has non-zero measure, so that our results allow us to make statements about the comodularity of $G \supset H^{x}=H^{x} \cap H^{x}$, the quotient space $G / H$ is discrete, and so $H$ is an open subgroup. In that case, it is already well-known (and trivial) that $\Delta_{G}(h)=\Delta_{H^{x}}(h)$ for all $h \in H^{x}$ and all $x$.

- If we consider the special case where $K=e$, we have $K^{y}=e$ for all $y \in G$, giving $s=e$. The conclusion from Corollary 1 in this case is trivial.

- If $H$ is a normal subgroup of $G$, then $H^{x}=H$ for all $x \in G$ and we have $\Delta_{H}(s)=\Delta_{G}(s)$ in consequence of the normality. Here, with an application of Fubini's Theorem (cf.[3], p.153), Corollary 1 becomes

$$
\Delta_{H^{x} \cap K^{y}}(s)=\Delta_{K^{y}}(s), \text { for } s \in H \cap K^{y} .
$$

But this is a fact anyway, since $H \cap K^{y}$ is normal in $K^{y}$.

Proof of Corollary 2. If $G \supset H$ is comodular, then so is $G \supset H^{x}$ for all $x \in G$. An application of Corollary 1 implies that $H^{x} \supset H^{x} \cap K^{y}$ is comodular for almost all $x$ and $y$. By conjugating with $y^{-1}$ and using Fubini's Theorem, it then follows by conjugation, that $K \supset H^{z} \cap K$ is comodular for almost all $z \in G$.

\section{REFERENCES}

[1] Gaal, Steven A.: Linear Analysis and Representation Theory. Springer, Berlin, (1973).

[2] Halmos, P.R.: The decomposition of measures. Duke Math. J.,Vol 8 (1941) p.386-392.

[3] Hewitt, E. and Ross, K.A.: Abstract Harmonic Analysis, Vol I, Springer, Berlin, (1963).

[4] Mackey, G.W.: Induced representations of locally compact groups I. Annals of Maths, Vol55, No1, (1952), p. 101-140.

[5] Reiter, Hans: Classical Harmonic Analysis and Locally Compact Groups. Oxford Mathematical Monographs, (1968).

Discipline of Mathematics, School of Physical Sciences, University of Tasmania, Tasmania 7001, Australia. E-mail address: kumudini@utas.edu.au

Building 12, Level 7, Room 20, School of Electrical and Computer Engineering, Rmit University Melbourne City Campus, Melbourne, Victoria 3001, Australia.

E-mail address: bill.moran@rmit.edu.au 